\title{
Capacidades humanas, democracia y Estado de bienestar habilitante: revitalización de la socialdemocracia en América Latina
}

\author{
L. Iván Valenzuela Espinoza \\ Universidad Arturo Prat, Iquique, Chile. \\ Email: leandroivalenzuela@gmail.com
}

\begin{abstract}
Resumen: El Estado de Bienestar Habilitante Nórdico ha sido propuesto como modalidad para proseguirel desarrollo de la socialdemocracia. Un rasgo clave de esta propuesta dice relación con el fortalecimiento de las capacidades humanas sobre la base de "servicios públicos cultores de capacidades". Esto se entrecruza sistémicamente con el desarrollo económico y tecnológico de una sociedad del conocimiento dinámica e innovadora, permitiendo hacer frente con éxito a nuevos desafíos y exigencias. La revisión teórica que lleva a cabo el presente artículo tiene como propósito poner al descubierto los rasgos centrales de las complejas dinámicas que concurren en el fenómeno indicado. Asimismo, contribuye a un abordaje teórico serio en torno a la relevancia para América Latina, en un sentido amplio, del Estado de Bienestar Habilitante Nórdico y su desarrollo de las capacidades humanas, y, de modo particular, a la revitalización de los debates socialdemócratas de la región.
\end{abstract}

Palabras claves: Capacidades, democracia, Estado de bienestar habilitante, socialdemocracia, teoría de la actividad.

\section{Human capabilities, democracy and enabling welfare state: revitalization of social democracy in Latin America}

\begin{abstract}
The Nordic Enabling Welfare State has been proposed as a modality for further development of social democracy. A key feature of this proposal relates to strengthening human capacities on the basis of "capacity cultivating public services". This intersects systemically with the economic and technological development of a society of dynamic and innovative knowledge, making it possible to successfully face new challenges and demands. The purpose of the theoretical review carried out in this article is to uncover the central features of the complex dynamics that concur in the indicated phenomenon. It also contributes to a serious theoretical approach to the relevance for Latin America, in a broad sense, of the Nordic Enabling Welfare State and its development of human capabilities, and, in particular, to the revitalization of social democratic debates in the region.
\end{abstract}

Keywords: Capabilities, democracy, enabling welfare State, social democracy, activity theory.

\section{Capacidades humanas, democracia e Estado de bem-estar habilitante: revitalização da social democracia na América Latina}

Resumo: O Estado de Bem-Estar Habilitante Nórdico foi proposto como uma modalidade para continuar o desenvolvimento da socialdemocracia. Uma característica fundamental desta proposta está relacionada ao fortalecimento das capacidades humanas baseadas em "serviços públicos geradores de capacidades”. Isto se vincula interseção sistematicamente com o desenvolvimento econômico e tecnológico de uma sociedade do conhecimento dinâmica e inovadora, permitindo-nos enfrentar com sucesso novos desafios e demandas. A revisão teórica que realiza o presente artigo tem como finalidade expor as características centrais das complexas dinâmicas que concorrem no fenômeno indicado. Contribui também para uma séria abordagem teórica da relevância para a América Latina, em um sentido amplo, do Estado de Bem- 
Estar Habilitante Nórdico e seu desenvolvimento das capacidades humanas e, em particular, para a revitalização dos debates socialdemocratas da região.

Palavras-chave: Capacidades, democracia, Estado de bem-estar habilitante, socialdemocracia, teoria da atividade.

\section{Introducción}

El complejo cariz que han tomado el desarrollo y la competitividad económica global de América Latina plantea la necesidad, entre otras, de avanzar en la elaboración de una nueva conceptualización y análisis teórico del protagonismo que desempeña la promoción y desarrollo de las capacidades humanas. Dicho protagonismo no se circunscribe únicamente a los ámbitos directamente asociados a la economía, sino que también remite a la educación, la capacitación y formación, el capital humano, las competencias, etc.

En la sociedad del conocimiento, la cual se abre paso con fuerza en América Latina, las instancias sociales volcadas a propiciar las capacidades humanas devienen en pilares para el desarrollo económico, tecnológico y productivo. Al mismo tiempo, las articulaciones entre el ámbito técnico-económico y el de las capacidades humanas, las cuales pueden ser propulsadas en instituciones y organizaciones específicas, hacen patente la necesidad de profundizar y expandir la participación ciudadana y su aprendizaje en la vida democrática.

Sin embargo, las interrelaciones mencionadas con frecuencia han sido escasamente investigadas y teorizadas en las discusiones académicas y de política pública asociadas(ver, por ejemplo, BID 2018; Brunner 2000; Busso, Bassi, Urzúa y Vargas, 2012). Se constata en este plano, por consiguiente, un desafío intelectual de gran envergadura tanto para el pensamiento económico-social latinoamericano como para el pedagógico-educativo. Urge sentar las bases para un diálogo teórico serio y sostenido entre ambas tradiciones intelectuales.

Por consiguiente, el presente artículo analiza críticamente argumentos e ideas centrales de diversas corrientes de pensamiento que giran en torno a las capacidades humanas. Paralelamente, se propone una alternativa teórica capaz de contribuir al abordaje interdisciplinario de las capacidades humanas, la educación, la tecnología y la economía. Asimismo, el artículo elucida los principios clave del llamado "Estado de Bienestar Habilitante Nórdico" en tanto que concreción institucional de alto nivel de las conexiones entre la economía, la tecnología y los "servicios públicos cultores de capacidades". Ahondar en estos desarrollos teóricos en curso permitirá enriquecer los actuales debates de la socialdemocracia en América Latina.

Numerosos estudios académicos han sido dedicados al pormenorizado análisis económico, social y político del desempeño que han tenido recientes gobiernos progresistas y de izquierda en América Latina (Edwards, 2010; Sader, 2016; Falletti y Paredo, 2017). Con toda seguridad, este conjunto de contribuciones académicas arrojará luz sobre críticas y atolladeros, así como sobre logros y posibles proyecciones de dichas experiencias políticas.

Varios de estos estudios, no obstante su valor, llevan a cabo análisis más bien de corte convencional de las políticas impulsadas por los gobiernos en cuestión. Esto significa que en los análisis aludidos a menudo no ha habido un tratamiento teórico profundo y sistemático del papel de las complejas relaciones entre, por una parte, las capacidades humanas y, por la otra, el desarrollo económico y tecnológico. El insuficiente trabajo teórico se manifiesta, además, en un inadecuado tratamiento del potencial papel protagonista del Estado en la promoción de las capacidades humanas en conexión con los requerimientos de la economía y la tecnología de la sociedad del conocimiento. Por tal motivo, en el presente artículo se efectúa una acuciosa revisión teórica del campo de estudio del Estado de Bienestar Habilitante Nórdico, a la vez que se propone un marco conceptual dado por el "enfoque sociocultural”, especialmente bajo su modalidad de "teoría de la actividad", el cual proporciona un adecuado y pertinente soporte analítico que da cuenta coherentemente de la articulación entre las capacidades humanas, la economía y la tecnología en un contexto social de profundización y expansión de la democracia. Asimismo, se redunda en el ámbito de la educación y la formación de capital humano en América Latina con el objetivo de auscultar posibles lecciones, aprendizajes e implicancias derivadas de la experiencia 
nórdica. Por último, y desde la teoría social, se arguye que el análisis llevado a cabo contribuye y enriquece los debates socialdemócratas en América Latina.

El presente artículo consta de cinco secciones. En la primera sección se presenta el tránsito del Consenso de Washington al Consenso Post-Washington, poniendo de relieve los rasgos distintivos del social liberalismo, la socialdemocracia y el populismo en América Latina. Se argumentará a favor de la necesidad de problematizar teóricamente y en términos de política pública en torno a las capacidades humanas en la perspectiva de una revitalización y renovación de la socialdemocracia. Por su parte, en la segunda sección se introduce la problemática de las capacidades humanas conforme al Estado de Bienestar Habilitante Nórdico en tanto que expresión política por excelencia de la socialdemocracia nórdica. En tal sentido, se postula que el "enfoque sociocultural", y en particular su vertiente de "teoría de la actividad", en un vasto marco de ciencia social interdisciplinaria, brinda las condiciones intelectuales apropiadas para servir como soporte analítico de cara a otras formulaciones académicas existentes sobre las capacidades. Además, se puntualiza que tal enfoque, por su talante democrático, es altamente compatible con la doctrina socialdemócrata. En este contexto, se presta especial atención a la educación. En suma, dicho soporte teórico alternativo redunda en valiosas posibilidades para la colaboración interdisciplinaria en los ámbitos de la economía, la tecnología, la educación y las capacidades humanas. Paralelamente, en la tercera sección se expone el Estado de Bienestar Habilitante Nórdico, afirmándose que éste cuenta con potencial para erigirse en una real alternativa al neoliberalismo en boga, dado sus notables logros en la consecución del "círculo virtuoso" entre educación y capital humano de alta calidad, democracia y desarrollo económico. La cuarta sección, de otra parte, se aboca al esfuerzo realizado en la teoría social por formular una nueva noción de libertad, a efectos de revitalizar la doctrina socialdemócrata y la democracia. De la mayor importancia, a este respecto, es la contribución del teórico social y filósofo Axel Honneth. Finalmente, en la quinta sección se lidia con consideraciones centrales en materia de educación, capital humano y capacidades humanas de acuerdo con desafíos económicos y tecnológicos que afectan a América Latina.

En definitiva, las eventuales lecciones y el aprendizaje que se derivan de un mejor abordaje teórico de las capacidades humanas, de la mano de una profunda comprensión de su papel en el Estado de Bienestar Habilitante Nórdico, suponen un importante potencial para enriquecer y revitalizar el pensamiento socialdemócrata en América Latina.

\section{El Consenso post-Washington, socialdemocracias y populismo}

\section{Social liberalismo, socialdemocracia y populismo}

De acuerdo con Sandbrook (2014,p.3), connotado estudioso de la socialdemocracia en los países en vías de desarrollo, diversas características de las sociedades latinoamericanas, y de índole político-económico internacional, han permitido en años recientes a diversos gobiernos de izquierda de la región impulsar políticas "heterodoxas". Entre estas especiales condiciones, distintos países de América Latina contaron con un mayor grado de autonomía, lo cual hizo posible disponer de mayores opciones para programas políticos de izquierda (Sandbrook, 2014).

Lo anterior se entrecruza, desde la década de 2000 en adelante, con el denominado "Consenso PostWashington” en tanto que giro político y económico por parte del Banco Mundial y otros agentes influyentes en términos del desarrollo (Evans, 2008).

Tras las limitaciones evidentes del neoliberalismo en el subcontinente y otras partes del mundo, el “fundamentalismo de libre mercado" (Block y Somers, 2014), bajo la modalidad de "Consenso de Washington", defendido durante décadas por el Banco Mundial y las agencias internacionales de desarrollo, fue progresivamente abriéndose paso la idea de tomar distancia con respecto a posturas extremas de políticas y orientarse hacia otras más flexibles y de corte centrista. Precisamente, el social liberalismo, especialmente bajo la versión de "Tercera Vía”, se erigió en soporte doctrinario central del mentado y nuevo "Consenso Post-Washington”. 
Entre otras características, dicho giro subrayó que los mercados libres requerían ser acompañados por instituciones apropiadas y por impulsos o políticas enfocadas a la promoción de las capacidades y libertades de los individuos. Una de las exposiciones más articuladas de este desarrollo conceptual del neoliberalismo, específicamente centrado en América Latina, es el de Sebastián Edwards (2010).

En su análisis, Sandbrook (2014, p.8) sostiene que el social liberalismo rescata la noción "positiva” de la libertad en una manifiesta opción por promover las capacidades humanas indispensables para el disfrute de las libertades. De este modo, el social liberalismo va más allá de la libertad "negativa” y su énfasis en la remoción de los pretendidos obstáculos del Estado al despliegue de la acción humana.

Con todo, Sandbrook (2014, p.9) arguye que el social liberalismo, a saber, la versión más sofisticada y actual del neoliberalismo, no supera sus diversas falencias, por lo que también ha fracasado como orientación de políticas económicas y sociales para un desarrollo social y ecológicamente sustentable.

En un afán por profundizar en el análisis de algunos gobiernos de centroizquierda en América Latina, Sandbrook (2014, p.10) propone conceptualizarlos como expresión de "socialdemocracia moderada" en tanto que estrategia política.

Esta estrategia política asume la realidad objetiva del neoliberalismo por lo menos en el corto plazo, al tiempo que ofrece garantías a los inversionistas mediante políticas fiscales y monetarias ortodoxas. La redistribución se busca mediante políticas desarrollistas del Estado, las cuales deberían incrementar el ingreso fiscal y la generación de empleo. Los derechos sociales de la ciudadanía ("ciudadanía social”) se reforzarían mediante protección social universal, transferencias y el mejoramiento de los servicios públicos. Tales gobiernos también fomentarían la participación ciudadana merced a cambios institucionales y legales pertinentes principalmente a nivel local.

De otra parte, la estrategia de la "socialdemocracia radical" para la consecución del socialismo hace hincapié en la redistribución no sólo del ingreso, sino que también de la riqueza y el poder en sus diversas expresiones. Como subraya Sandbrook (2014), las dificultades para esta estrategia en uno o sólo un puñado de países son descomunales, dado el contexto global que le es más bien adverso. En tal perspectiva, tales experiencias de avance hacia el socialismo requieren contar con un bloque subcontinental poderoso para sobrevivir y prosperar en el tiempo. Esta estrategia enfatiza que pese a las adversidades, únicamente los medios democráticos han de ser utilizados y desarrollados en la contienda política.

Por último, Sandbrook (2014) constata la aparición de un "populismo de izquierda” en algunos países con una larga tradición histórica de populismo clásico. Sin embargo, el nuevo tipo de populismo en cuestión sería inequívocamente de izquierda, a la vez que adoptaría procedimientos y enfoques democráticos en su afán por avanzar en igualdad, y derechos sociales, en una apuesta de ruptura con el neoliberalismo.

\section{Capacidades humanas: propuestas teóricas}

Con la finalidad de contribuir intelectualmente a los debates en curso sobre el desarrollo en América Latina, propongo que se dé inicio a una reconceptualización sostenida y rigurosa de los estudios diversos sobre las capacidades humanas.

Así entonces, como se verá más adelante, la socialdemocracia nórdica logró resultados simplemente notables en materia de desarrollo económico y social, al tiempo que sentó bases sólidas para la innovación y la sociedad del conocimiento (Block, 2011). Por supuesto, estas sociedades están muy lejos de ser perfectas, por lo que también han de lidiar con serios problemas de diversa índole, sobresaliendo, entre otros, el incremento de la pobreza y la desigualdad, las presiones fiscales y la inclusión de la población migrante (Block, 2011).

Pues bien, a diferencia de los análisis convencionales, en el presente artículo se pone el acento en el papel decisivo que cumplen los servicios públicos y las políticas que cultivan y desarrollan las capacidades 
humanas. Sostengo que las diversas corrientes de la socialdemocracia y la izquierda latinoamericana pueden beneficiarse considerablemente de una mayor comprensión y conocimiento del enfoque en cuestión.

En tanto que encuadre inicial de la problemática de las capacidades, se debe indicar taxativamente que el neoliberalismo, en su expansión global, se ha entrecruzado, de manera en sumo compleja, con corrientes de pensamiento gerencial que han puesto el acento en términos tales como capital humano, competencias, emprendimiento, educación continua, aprendizaje de por vida, etc. (Sum y Jessop, 2015). Conforme se ha desarrollado el neoliberalismo, éste ha hecho una reducción de complejidad mediante la selección de términos, vocabularios, conceptos, etc., que dicen directa relación con las capacidades humanas (Sum y Jessop, 2015).

\section{Capital humano y enfoque de capacidades}

En décadas recientes, el neoliberalismo propició el concepto de capital humano reforzando el tratamiento conceptual de la economía como dominio analíticamente separado de la sociedad, y que puede ser entendido cabalmente a partir de sus rasgos distintivos internos. Este abordaje adopta como supuesto que los individuos emprenden acciones racionales con el objetivo de maximizar sus preferencias, a saber, calculando la relación entre costos y beneficios en la búsqueda de su interés propio. En los hechos, dado su andamiaje conceptual, esta peculiar concepción del comportamiento humano, en suma, prescinde de una comprensión acabada de cualquier otra actividad humana que no sea la que se manifiesta en el intercambio de mercancías en el contexto del mercado capitalista.

Por consiguiente, la noción economicista de capital humano no ha logrado asentarse como modelo explicativo adecuado para el desarrollo de políticas públicas volcadas al fortalecimiento de las capacidades, ya que no es capaz de reconocer diferencias cualitativas existentes entre conjuntos de instituciones y organizaciones comprometidas con el desarrollo de las capacidades humanas. De manera notoria, el enfoque de capital humano suele pasar por alto determinadas capacidades, tales como la creatividad y la imaginación, que son indispensables precisamente en contextos de intenso cambio e innovación social y tecno-económica (Miettinen, 2013; 2014).

De manera muy ilustrativa, el enfoque de capital humano en el ámbito de la educación ha hecho intentos recientes (Carneiro y Heckman, 2003) por ampliar su abordaje mediante la incorporación parcial de la posición social y los recursos culturales de las familias como fuente de desarrollo cognitivo, al tiempo que ha dado prioridad a intervenciones en la temprana infancia y en servicios personalizados para estudiantes en dificultades, en lugar de inversiones generales en los sistemas educacionales. Dicha aproximación afirma acertadamente que las intervenciones en la temprana infancia para los niños desventajados son más efectivas que intervenciones en momentos posteriores de la vida.

Paralelamente, y específicamente en los estudios económicos del desarrollo, Sen (1984) puso en marcha el "enfoque de capacidades".

Este enfoque tiene como objetivo brindar una versión más integral y sofisticada del desarrollo y las capacidades humanas que las existentes en formulaciones economicistas extremas. Con todo, Sen intenta llevar a cabo esta reformulación del desarrollo y las capacidades desde el liberalismo político y económico. Así entonces, la libertad de los individuos sólo puede desplegarse cabalmente en la medida que se sustente activamente en capacidades tales como la alfabetización y el acceso a educación de alta calidad. En otras palabras, es necesario que las políticas públicas definan y promuevan un conjunto de capacidades humanas intrínsecamente valiosas, las cuales, en verdad, conforman las bases mismas del bienestar y el desarrollo. Estas capacidades recomendadas por Sen pueden ser entendidas como los derechos y las libertades positivas de los individuos. Debe subrayarse que las ideas del autor en cuestión inspiraron la formulación del índice de Desarrollo Humano de Naciones Unidas (PNUD), el cual mide el desarrollo humano incluyendo la educación y la salud, por lo que supera notablemente los estrechos límites de los indicadores estrictamente economicistas.

Empero, la teoría de Sen sigue siendo la de un economista liberal al momento de definir, en el fondo, las 
capacidadesen tanto que capacidad en el uso y manipulación de mercancías y bienes de consumo. En definitiva, y desde la perspectiva de la política pública, lo que cuenta en verdad es lo que el individuo puede hacer y ser con recursos a su disposición, constatándose diferencias en las capacidades de hacer y ser a partir de una misma canasta de mercancías o monto monetario disponible. Por lo mismo, las derivaciones a partir del enfoque de capacidades corren el riesgo de no estar en sintonía con formas y expresiones socioculturales no comerciales, comprendiendo tradiciones locales, educación pública, entre otras, además del carácter público y culturalmente compartido de la ciencia y el conocimiento profesional (Miettinen, 2013, p.159). En la medida que el enfoque en cuestión no es capaz de problematizar profundamente acerca de las capacidades humanas en circunstancias y condiciones ajenas a las del funcionamiento de la lógica mercantil, éste no puede más que trastabillar teóricamente, derivando irremediablemente en una formulación más bien empobrecida de las auténticas fuentes del desarrollo de las capacidades humanas.

En suma, las elaboraciones teóricas en torno a las capacidades hechas desde premisas liberales e individualistas no proporcionan bases sólidas en esta materia. Resulta necesario, por lo tanto, prestar la mayor atención a intentos serios por desarrollar un enfoque alternativo.

\section{El enfoque sociocultural de las capacidades humanas: aprendizaje, educación y democracia}

Un desarrollo intelectual interdisciplinario en curso tanto en las ciencias sociales como en las educacionales, y que ofrece una línea alternativa crítica de investigación sobre las capacidades humanas, es el enfoque sociocultural fundado, en la década de 1920, por el psicólogo soviético L. S. Vygotsky (1987). Dado sus orígenes en el pensamiento de Marx, este enfoque parte del supuesto que las capacidades humanas, entre ellas, y de manera crucial, el aprendizaje, sólo pueden ser teorizadas adecuadamente si son arraigadas y relacionadas con sendos procesos socio-históricos en los que se desenvuelve la interacción sociocultural. De acuerdo con el enfoque sociocultural, las mentes individuales y el desarrollo humano de capacidades emergen, se despliegan y empoderan en complejas interacciones y relaciones sociales, las cuales entrañan modalidades compartidas de actividades materiales, prácticas e intelectuales. De manera más específica, Vygotsky es el fundador de la "teoría de la actividad histórico-cultural”.

Así pues, el enfoque sociocultural hace hincapié en que las capacidades y el aprendizaje están en el corazón mismo del desarrollo integral de la personalidad y la creatividad, entroncando de manera explícita y directa con el concepto Bildung de insignes pensadores sociales, filósofos y pedagogos alemanes a caballo entre los siglos XVIII y XIX (Good, 2005; 2018). En síntesis, Bildung comprende la autorrealización y crecimiento del individuo, así como el desarrollo de sus capacidades e identidad mediante su participación, crítica y contribución a la cultura de la que es miembro activo. Por lo mismo, esta noción no se circunscribe a la mera educación formal y al desarrollo cognitivo.

En tal perspectiva, Good (2018) resalta tres dimensiones en el concepto de Bildung. En primer lugar, conforme a este entendimiento, el individuo encuentra su vocación singular para lo que es apto en la medida que contribuye al desarrollo de su propia cultura, generándose una interdependencia positiva entre el individuo y su sociedad. En segundo lugar, la noción en cuestión sugiere que una variedad amplia de experiencias es indispensable para desarrollar una mente abierta e inteligente. En términos pedagógicos esto implica despertar en los niños y adolescentes múltiples intereses. Finalmente, el desarrollo de las capacidades de los individuos y su vocación comprenden no solamente una modalidad única de participación sociocultural, sino que también un pensamiento crítico e independiente que es de especial importancia para el desarrollo posterior de dicha cultura.

El abordaje sociocultural acerca del desarrollo y el aprendizaje humano propone encaminar las políticas de fomento de las capacidades desde las habilidades cognitivas hacia el desarrollo del individuo en tanto que ciudadano que es participante en un campo cultural y miembro de la humanidad. El aspecto medular para la educación debería ser el desarrollo de la vocación del individuo para contribuir a la sociedad democrática, y convertirse en un integrante reconocido de la misma. La identificación y apoyo a expresiones tempranas de 
talentos específicos e intereses constituye probablemente la "dimensión más sutil y exigente del trabajo pedagógico” (Miettinen, 2013, p.164).

Ahora bien, lo expuesto se refuerza y complementa con la valiosa contribución al debate sobre la "libertad social” (Honneth, 2017, p.24; p.25) del teórico social Axel Honneth. Según este punto de vista, la participación y cooperación en la vida de la comunidad no sólo es una condición indispensable para la libertad, sino que también su soporte decisivo. Elaborando a partir Hegel, Marx y Dewey, el argumento de Honneth redunda en que la libertad es lisa y llanamente inseparable de la experiencia gratificante que hace posible el desarrollo del sujeto individual, proceso en el que éste descubre sus capacidades y talentos con los cuales, a su vez, contribuye a su sociedad y cultura mediante instituciones tales como la división del trabajo y las asociaciones profesionales.

El planteamiento de Honneth (2017, p.60-63) prosigue, en lo medular, llamando la atención sobre el hecho que la erradicación de formas de exclusión entraña la eliminación de barreras a la comunicación y a la interacción social dentro comunidades insertas en sociedades democráticas. Estas condiciones, asimismo, hacen posible avanzar en mayores niveles de reconocimiento mutuo de los grupos sociales. Por último, la elaboración de estas ideas permite dotar de bases histórico-institucionales a la libertad social, así como una orientación preliminar a una “comprensión experimental del socialismo".

\section{Estado de bienestar habilitante}

\section{Las tres modalidades}

El foco en el empoderamiento humano mediante el despliegue efectivo de las capacidades, fenómeno al que se ha hecho permanente referencia arriba, se expresa de manera explícita y nítida en la noción de "Estado de Bienestar Habilitante” y sus tres modalidades centrales (Miettinen, 2013).

En primer lugar, cabe mencionar la versión neoliberal de Gilbert (2004;2005), quien hace hincapié en la necesidad de privatizar los servicios públicos, la adopción de criterios de mercado en el ámbito estatal, así como en la preferencia por la focalización selectiva en lugar de coberturas universales. Las asociaciones voluntarias de la sociedad civil y las empresas deberían asumir la provisión de los servicios que tradicionalmente han quedado bajo la responsabilidad del Estado.

De otro lado, Curtis (2006) ofrece una propuesta social liberal de "Estado Bienestar Habilitante”, conforme a la cual, éste, en funciones de "primer orden", se centra en la mantención de la infraestructura, la inversión social permitida por el presupuesto público, así como la estabilidad jurídico-legal. Tales funciones se sustentan operacionalmente en acciones de mando, focalización, evaluación de desempeño, regulaciones, etc. De acuerdo con este abordaje, no cabe albergar grandes ilusiones con respecto a este "primer orden" dado su sesgo al control jerárquico y rigideces asociadas. Por su parte, las funciones de "segundo orden" en el papel habilitante de Estado remiten a la experimentación y al aprendizaje, en el entendido de que ello hace posible la redefinición permanente de estándares y metas compartidas, a la vez que refuerza el diálogo estatal con actores varios del mercado y la sociedad civil. Esto último, por cierto, es altamente valorado por el autor.

Finalmente, resultante altamente interesante la versión del Estado de Bienestar Habilitante desarrollada por el pragmatismo institucional (Sabel, 2006; Herrigel, 2007; Kristensen y Lilja, 2011).Conforme a este enfoque, la provisión de servicios públicos crecientemente debe ser personalizada y ajustada a las diferentes e individuales necesidades de usuarios y clientes. En tal perspectiva, se propende a la exploración creativa de condiciones institucionales y organizacionales que propicien la convergencia entre sendos procesos de "aprendizaje institucional", la mejora continua de los servicios públicos y la participación en clave de "experimentalismo democrático" (Dorf y Sabel, 1998). Asimismo, el Estado de Bienestar Habilitante ha de contar con dispositivos institucionales que efectivamente logren que los actores compartan sus respectivos conocimientos especialmente al tratarse de problemas compartidos más ampliamente. A este respecto, las innovaciones organizacionales en empresas post-fordistas, tales como Toyota, serían ilustrativas de formas de aprendizaje sustentadas en experimentaciones institucionales (Sabel, 2006). 
Se debe consignar que la conceptualización actual en torno al Estado de Bienestar Habilitante es altamente compatible con el indispensable trabajo realizado sobre el particular por Esping-Andersen $(1990,2002)$ y Mjoset (2011), entre otros connotados especialistas. Al primero debemos, en una apretada síntesis, la formulación de una influyente tipología de Estados de Bienestar, incluido, cómo no, el nórdico. En este análisis se ponen de manifiesto los rasgos que diferencian al sistema nórdico de bienestar de los existentes en otras sociedades del mundo desarrollado. Paralelamente, Esping-Andersen ha sido uno de los pioneros al resaltar la importancia de la política social en la temprana infancia, y el correspondiente entorno familiar, en el fomento de condiciones iniciales equitativas y justas de desarrollo para la niñez más allá de su origen de clase y grupo social. Por su parte, Mjoset (2011)ha impulsado la investigación comparada entre las sociedades nórdicas con un fuerte énfasis en sus dotaciones económicas e institucionales de cara a los desafíos de la innovación.

Pues bien, a partir de los principios vistos arriba sobre el Estado de Bienestar Habilitante, Reijo Miettinen (2011, 2013, 2016), destacado teórico finlandés sobre las relaciones entre capacidades humanas, democracia y políticas públicas, ha elaborado una propuesta de actualización y nuevo desarrollo para el "Estado de Bienestar Habilitante Nórdico" (EBHN)acorde con diversos retos contemporáneos. La propuesta de Miettinense sitúa inequívocamente en un intento de reconceptualización del EBN en consonancia con la tradición socialdemócrata nórdica y las diversas exigencias del mundo actual, y, por consiguiente, adopta como punto de partida los notables logros de las políticas públicas y sociales alcanzados por los sistemas democráticos de Dinamarca, Finlandia, Suecia y Noruega (Block, 2011; Esping-Andersen 1990, 2002; Mjoset 2011).

A juicio de Miettinen (2013,p.175; p.177),el modelo nórdico, en primer lugar, se distingue, entre otros modelos europeos de bienestar, por su énfasis en la provisión de servicios públicos de alta calidad para brindar igualdad de oportunidades en vez de la transferencia directa de dinero para hace frente a los riesgos sociales. Por lo tanto, los países nórdicos están a la vanguardia en materia de construcción de un Estado de Bienestar sobre la base de los servicios tales como jardines infantiles, educación, salud infantil y materna, bibliotecas públicas, cultura, entendidos como servicios públicos universales que son clave en tanto que instituciones cultoras de capacidades humanas y enriquecedoras de la vida sociocultural de la democracia.

De la mayor importancia resulta ser la educación finlandesa, ya que ésta da cuenta, entre otros aspectos, de un sistema centrado en necesidades educativas especiales que busca "la provisión de servicios habilitantes individualizados” (Miettinen, 2013, p.175). Sin embargo, la individualización de la educación no tiene lugar a través del mercado, sino que a través de un sistema educativo público y gratuito.

En segundo lugar, en las décadas de 1970 y 1980 se crearon servicios públicos de la mano de la formación de equipos de profesionales a cargo de los mismos, al tiempo que se fomentó la investigación concomitante para su constante desarrollo. Por lo mismo, entre los servicios públicos y las "comunidades multi-profesionales” en cuestión conforman, tras el paso del tiempo, interactivos e híbridos "campos multi-organizacionales” de los que crecientemente participan usuarios, clientes y asociaciones de ciudadanos. El consiguiente aprendizaje institucional y horizontal es la piedra angular tanto para el mejoramiento y desarrollo de la calidad de los servicios públicos como para la participación democrática de la ciudadanía.

En el caso de la educación finlandesa, por ejemplo, el "campo-multi-organizacional" de escuelas y profesores se conecta sistémicamente con la investigación pedagógica que se lleva a cabo en las universidades. Esto es de la mayor importancia para la educación continua de los equipos directivos, los docentes y, por último, para el desarrollo de remediales, materiales didácticos y soluciones pedagógicas ajustadas a las necesidades locales de los estudiantes (Miettinen, 2013).

En tercer lugar, el sistema educacional nórdico y otros servicios públicos han permitido un notable incremento en la población del nivel y calidad de sus estudios, escolarización y formación (Esping-Andersen 1990, 2002). En palabras de Miettinen (2013, p.176)

“una población bien educada proporciona un tremendo potencial de conocimiento práctico, profesional y teórico distribuido en todas las esferas de la sociedad. Esto constituye una base ampliada para la 
actividad innovadora. También subraya la necesidad de democratización de la innovación, y cuestiona la naturaleza elitista de la innovación”.

En cuarto lugar, por su parte, cabe poner de relieve la tradición histórica del mundo nórdico en términos de descentralización a nivel local y municipal, lo cual contribuyó de sobremanera a la respectiva descentralización de los servicios públicos universales del primer Estado de Bienestar Nórdico y a sus lazos con instituciones, valores y prácticas democráticas (Block, 2011).

En quinto lugar, en perspectiva comparada, los ciudadanos nórdicos suelen ser activos integrantes de asociaciones varias, lo cual es de incalculable valor para el empoderamiento de los individuos y agrupaciones de la sociedad civil. Dice Miettinen (2013, p.177), "tanto la tradición de democracia local como la participación activa de los ciudadanos en asociaciones constituye una base para la profundización y ampliación de la democracia en la producción e innovación de los servicios y la política”.

Finalmente, la idea del Estado de Bienestar Habilitante cabe ser entendida como una elaboración del "círculo virtuoso" de igualdad, desarrollo económico y democracia, el cual se encuentra en el corazón mismo del ideario y doctrina socialdemócrata nórdica, y en la concreción y realización histórico-institucional y política del Estado de Bienestar Nórdico (Miettinen, 2013; Mjoset 2011). De hecho, tales ideas caracterizaron las alusiones del destacado economista sueco, Gunnar Myrdal, al despliegue de la creatividad de la ciudadanía (Kettunen, 2012).

\section{Democracia, libertad social y experimentación}

\section{En torno a la libertad social}

El notable pensador socialista húngaro Karl Polanyi (2001), hace décadas puso de manifiesto que la noción de libertad neoclásica redunda en una versión excesivamente individualista para las necesidades de una sociedad compleja y democrática, razón por la que se tornaba perentorio desarrollar una noción distinta y alternativa de libertad.

Como se ha señalado arriba, la libertad concebida como "máxima autonomía individual" simplemente no brinda base sólida para la conjunción de la libertad del sujeto individual con la comunidad social. No obstante, tampoco ha resultado viableel colectivismo uniformador que pierde de vista el despliegue de las diferencias individuales. Por lo mismo, Axel Honneth (2017), en línea con las nuevas interpretaciones de la filosofía y teoría social de Hegel, propone vertebrar una renovada "posición intermedia" de libertad, la que, de hecho, se inspira, al igual que el mencionado llamado de K. Polanyi ${ }^{1}$,en el hallazgo clave del socialismo temprano o "utópico" (Honneth, 2017, p.28). De acuerdo con esta conceptualización (Honneth, 2017, p.28-29), "los sujetos individuales sólo pueden realizar su capacidad para la libertad en tanto que miembros de una comunidad social libre". En este tipo de comunidad el cumplimiento recíproco de "intenciones compartidas generalmente” tiene lugar sin coerción en contextos de interacción y comunicación que propician la valoración, la cooperación y la consideración mutua.

Es menester poner énfasis en que la redefinición de la libertad individual de Honneth (2017, p.28) conlleva que ésta sea dependiente de otros sujetos, con lo cual deviene en "libertad social”, la que, por su parte, se constituye en un "elemento teórico del individualismo holístico". Este último se plantea como posición "socio-ontológica" que afirma que la realización de ciertas capacidades humanas requiere la existencia de comunidades sociales y, por ende, de entidades holísticas, pero sin extraer la conclusión de que los sujetos individuales como tales sean incompletos o "incluso no existentes".

A efectos de aclarar sus premisas teóricas, Honneth (2017, p.19) hace alusión a dos modelos de comunidades sociales en la perspectiva de diferenciarlos según las formas que adquieren las metas y propósitos de la acción humana en ellas. El paso conceptual dado por Honneth, en este aspecto, engarza con la tradición de pensamiento crítico inaugurada por $\mathrm{Marx}^{2}$. De acuerdo con el primer modelo, los individuos persiguen metas 
compartidas que pueden lograr unitariamente sin que sea necesario que intencionalmente persigan las mismas de manera unitaria. La economía de libre mercado con su mecanismo de "mano invisible" da cuenta de este tipo de "realización colectiva de metas". Según esta lógica económica, como es sabido, los individuos persiguen sus intereses económicos, al tiempo que contribuyen al bienestar general. En el segundo modelo, por contra, las "metas entrecruzadas demandan que los miembros de la sociedad hagan de estas metas máxima o propósito de sus propias acciones individuales". En este caso no sólo hay solapamiento o superposición entre los individuos al actuar entre sí, sino que, además, estos actúan "para cada uno" con intenciones conscientes para conseguir "propósitos compartidos de forma común”.

El giro hacia el principio de la "libertad social" expuesto arriba, hace posible, en sociedades complejas y diferenciadas funcionalmente, relacionarlo con la formación y desarrollo de la voluntad democrática en el marco de la actividad política.

A juicio de Honneth (2017, p.82), el economicismo, que ha tendido a caracterizar a los enfoques teóricos del socialismo, se tradujo en una ausencia fatal de valoración del potencial emancipatorio de la formación de voluntad democrática y deliberación en la esfera política. Dicho de otro modo, el gran hallazgo del socialismo temprano de la "libertad social" únicamente fue visualizado en el dominio de economía, razón por la que se confirió prioridad a la transformación radical dentro de la misma, ignorándose de plano su potencial emancipatorio para la política, las libertades civiles y políticas, la familia y las relaciones de género(2017,p.82;p.85;p.86).Lisa y llanamente esta falencia no se condecía con la alta complejidad y diferenciación funcional de la sociedad contemporánea ${ }^{3}$ (Luhmann, 1995; Jessop, 1990). Por lo mismo, y en palabras de Honneth (2017, p.88), "si el socialismo aún pretende representar la visión de una mejor forma de vida, entonces demos definir cómo estas esferas independientes de la libertad social han de relacionarse adecuadamente entre sí en el futuro”.

El enfoque de Honneth (2017, p.96) hace directa referencia al pensamiento de Dewey sobre la experimentación social a fin de reducir o eliminar las barreras a la comunicación y la interacción en las diferentes esferas sociales, apuntalando de este modo el reconocimiento entre los diferentes actores sociales.

Precisamente, el desarrollo de los servicios públicos cultores de capacidades humanas, como se ha visto, en el contexto del Estado de Bienestar Habilitante Nórdico, supone un acicate para avanzar en las condiciones institucionales requeridas para el despliegue de la libertad social y la expansión de las posibilidades de la democracia.

\section{Estado de bienestar habilitante nórdico y la nueva agenda de la socialdemocracia en América Latina}

En los campos multi-organizacionales del Estado de Bienestar Habilitante Nórdico vistos arriba se dan citan conjuntos de instituciones constituidos por servicios públicos que promueven las capacidades humanas generando y empoderando distintas modalidades de agencia individual y colectiva.

En tanto que rasgo clave del creciente protagonismo social de la educación y la formación en varios países de América Latina, los respectivos conjuntos de instituciones y servicios públicos, en su existencia concreta, pueden ser concebidos en tanto que "campos multi-organizacionales" (escuelas, universidades estatales y privadas, institutos de formación profesional, centros de investigación, etc.) de alta complejidad.

Entre los numerosos desafíos de capacidades humanas que enfrenta América Latina despuntan, entre otros, los procesos de digitalización y automatización que podrían afectar a dos tercios de los ocupados en algunos de sus países (BID, 2018, p. 92). Así entonces, la robotización y automatización en el mundo del trabajo suponen una seria amenaza para la empleabilidad de millones de trabajadores, empleados y profesionales de la región. El uso intensivo y eficiente de las nuevas tecnologías en la producción y los servicios demandará un amplio y diverso abanico de competencias y habilidades, todo lo cual está poniendo a prueba a los sistemas de educación y de formación de capital humano y capacidades humanas. Estudios recientes ponen de manifiesto que en América Latina ya está teniendo lugar la desaparición de numerosas labores rutinarias o su sustitución por alternativas tecnológicas (BID, 2018, p. 93). 
Tanto en el ámbito académico como en el de la política pública de América Latina se ha constatado que la forma apropiada para encarar el desafío señalado es a través de la formación de competencias y capacidades, y que la educación debe modernizarse profundamente para estar a la altura de los requerimientos de la sociedad del conocimiento (ver, por ejemplo, Brunner, 2000; Busso et al. 2012).

Los nuevos requerimientos del mercado laboral y la sociedad en general son destacados en el "Programa Internacional de Evaluación de los Alumnos”(PISA) de la Organización para la Cooperación y el Desarrollo Económicos (OCDE). Cabe subrayar que México y Chile ya integran dicha organización y que Colombia prontamente hará la mismo. Pues bien, la medición que hace la prueba PISA no sólo evalúa el conocimiento, las competencias y las habilidades de los estudiantes que están próximos a finalizar su educación obligatoria, sino que también pretende poner al descubierto la capacidad de su aplicación al problemas y situaciones del mundo real. Los resultados en América Latina hacen patente la necesidad imperiosa de modernizar profundamente su sistema educativo. A modo de ilustración, se constata que uno de cada cuatro estudiantes de 15 años es incapaz de encontrar una o más secciones independientes de información explícitamente indicadas, de reconocer el tema principal o propósito de un autor en determinado texto o tema conocido, y de relacionar coherentemente entre sí textos y conocimiento cotidiano (Busso et al., 2012 p, 65).

La situación es aún más alarmante en lo que respecta a las competencias matemáticas, ya que el 35\% de los estudiantes es incapaz de identificar información y realizar procedimientos matemáticos rutinarios. En otras palabras, uno de cada tres estudiantes simplemente no está en condiciones de responder preguntas aparentemente obvias y claramente definidas, pese a disponer de toda la información necesaria y pertinente para la resolución de los problemas planteados (Busso et al, 2012 p, 65).

Por su parte, el Programa para la Evaluación Internacional de Competencias de Adultos (PIAAC), congruente con los lineamientos de la OCDE, pone el acento en la urgencia de asir cabalmente que la comprensión lectora, el razonamiento matemático y la resolución de problemas son "habilidades clave para el procesamiento de información”, siendo indispensables para la participación en el mercado laboral, la educación, la capacitación y la vida social(OCDE, 2017, p. 62).

A modo de ilustración de las falencias generalizadas en América Latina en términos de la formación de capacidades humanas, en Chile, apenas un 11,9\% de los adultos alcanza un nivel $3^{4}$ o superior en razonamiento matemático, a la vez que un magro 14,5\% logra este nivel en comprensión lectora. Ambas cifras se ubican muy por debajo de los promedios OCDE, a saber, un 43\% en razonamiento matemático y 46\% en comprensión lectora (OCDE, 2017, p. 63). Asimismo, sólo un 15\% de los adultos chilenos obtiene el nivel 2 o 3 en la resolución de problemas en entornos informáticos, situándose nuevamente por debajo 31 \% promedio de la OCDE(OCDE, 2017, p. 63).

Con todo, pese a los profusos llamados, en el contexto latinoamericano, a efectuar cambios profundos en la educación y de formación de capacidades humanas, persiste la insuficiente teorización acerca de cómo éstas se relacionan de forma compleja con el desarrollo de la economía y la tecnología en la sociedad del conocimiento. Tal falencia se comprueba en el ámbito académico como en el de la política pública (ver, por ejemplo, Brunner 2002; Busso et al. 2012; BID 2018). Por lo mismo, resulta altamente relevante para el problema en cuestión ahondar en las conceptualizaciones y metodologías que están en la base de los notables logros de los países nórdicos en materia de capacidades humanas y desarrollo tecno-económico. Como se mostrado arriba, el enfoque sociocultural proporciona herramientas conceptuales y metodológicas para la investigación de cómo potenciar los diferentes "campos” institucionales y organizacionales que dan impulso sostenido y sistemático a las capacidades humanas y al capital humano de alto nivel.

Así entonces, conforme al enfoque sociocultural, y en una apretada síntesis, el estudio de dichos campos ha de proceder de la siguiente manera (Miettinen, 2013, p.114-116). En primer lugar, es menester conocer y desentrañar las interacciones que al interior de los campos producen aprendizaje institucional por medio de la actividad que despliegan los equipos multi-profesionales en distintos niveles. A su vez, la teoría de la actividad, dado su origen en la dialéctica, estipula que el desarrollo de los campos obedecen a sendas contradicciones que se expresan en tanto que problemas por resolver, los cuales comprenden tensiones y dilemas concomitantes. En 
tercer lugar, la investigación debe poner al descubierto cómo las soluciones adoptadas se manifiestan en el desarrollo de nuevas herramientas intelectuales y materiales que dan cuenta del aprendizaje, y de la "remediación" asociada, de los actores sociales dentro del campo. Lo anterior pone en marcha sendos cambios y transformaciones en las prácticas sociales. Paralelamente, el cambio de las prácticas sociales va acompañado de producción y difusión de nuevo conocimiento al interior del campo multi-organizacional.

La compleja dinámica esbozada arriba da cuenta de un proceso de "aprendizaje expansivo", conforme a la "tercera generación"5 de la teoría de la actividad de Engeström (1987). Según este planteamiento, estamos frente a un aprendizaje expansivo cuando el conjunto de la actividad es transformado de manera significativa a la luz de la activa participación de los actores involucrados en la misma.

Por consiguiente, el aprendizaje expansivo en las actividades de los campos multi-organizacionales conlleva el despliegue de las capacidades humanas mediante el cuestionamiento, la identificación de problemas y la propuesta de soluciones y remediales según las necesidades locales y situacionales. Esto significa la posibilidad real de poner en marcha nuevas transformaciones en diversos espacios sociales e institucionales que conllevan la expansión y profundización de la participación democrática de la ciudadanía. El aprendizaje expansivo, por tanto, abre sugerentes posibilidades para potenciar mediante la agencia humana los lazos entre la libertad social y la profundización democrática.

La sociedad del conocimiento en América Latina plantea en sus más diferentes ámbitos la necesidad de desarrollar instituciones, organizaciones y prácticas sociales que apuntalen mayores niveles de aprendizaje y experimentación social en la vida democrática. Ésta es una lección fundamental que se deriva de la experiencia nórdica de Estado de Bienestar Habilitante. Por lo mismo:

"el aprendizaje institucional en asociaciones de desarrollo y campos multi-organizacionales es el prerrequisito básico de un Estado habilitante. En la teoría pragmatista éste también es clave para la profundización de la democracia. Las personas son capaces de desarrollar sus capacidades mediante la participación en la solución de problemas sociales por parte de comunidades de desarrollo” (Miettinen, 2013, p.186).

En línea con su visión de exploración experimental de soluciones, Dewey estimaba que la democracia remitía a las interacciones delos individuos, la comunidad y el Estado. De esta manera, los derechos positivos de los individuos para desarrollar sus capacidades dependen de cómo se organizan el mundo del trabajo, la vida comunitaria y el Estado. Así entonces, y a juicio de Evans, (2000), para el pensador norteamericano la democracia está íntimamente conectada con "el concepto de igualdad definida como la libertad generada por la sociedad para que los individuos desarrollen plenamente el potencial de cada uno para la participación en la vida común de todos".

El modelo de Dewey de resolución de problemas mediante la experimentación social conlleva componentes de investigación, aprendizaje reflexivo y reconstrucción crítica de las prácticas sociales (Campbell, 1992; Burke, 1994). En otras palabras, cuando las formas habituales y acostumbradas no funcionan o se enfrenta un problema social, se torna indispensable reflexionar sobre las condiciones de la acción. A resultas de lo anterior se genera una hipótesis de trabajo para cambiar la situación y proporcionar una solución. Los intentos por implementar la solución finalmente ponen a prueba la hipótesis de trabajo, con lo cual se evalúa el éxito o no del experimento.

Las modalidades conceptuales y metodológicas señaladas refuerzan los vínculos entre el mejoramiento de la democracia, el despliegue de las capacidades humanas y el desarrollo de instituciones y organizaciones que permitan afrontar los desafíos económicos y tecnológicos actuales. Una configuración específica de lo anterior es el Estado de Bienestar Habilitante Nórdico. Su conocimiento serio y profundo pone al descubierto principios, conceptos y abordajes susceptibles de enriquecer la reflexión y los debates de la socialdemocracia en América Latina. 


\title{
Conclusión
}

Los rasgos centrales y principios del Estado de Bienestar Habilitante Nórdico puestos al descubierto arriba hacen posible, de la mano de esfuerzos teóricos y metodológicos, sugerir ideas y conceptos útiles en América Latina en materia de qué tipo de conocimientos y medidas impulsan y promueven el desarrollo de las capacidades humanas a tono con los desafíos de la economía y la democracia. De manera específica, la teoría de la actividad brinda un valioso basamento conceptual interdisciplinario para investigar y avanzar en el conocimiento de la problemática señalada. También se ha mostrado cómo el abordaje de las capacidades humanas, a la luz de la experiencia nórdica, supone una notable fuente de ideas, y conceptos novedosos, para la revitalización de los debates de la socialdemocracia en América Latina, y la formulación de alternativas viables al neoliberalismo.

\section{Notas}

\begin{abstract}
${ }^{1}$ La tradición intelectual que sigue las directrices conceptuales de Karl Polanyi ha generado una valiosa contribución a la teoría social y al acervo teórico del socialismo democrático. Muchos argumentos de esta tradición son altamente compatibles con ideas centrales de Honneth (2017). Ver, por ejemplo, Block (2011, 2014), Evans (1995) y R. Sandbrook (2006; 2014).

${ }^{2}$ La teoría de la actividad histórico-cultural, dado sus orígenes en el pensamiento de Marx, ha elaborado en sus distintas modalidades en torno al potencial del "ser genérico" humano para transformar mediante sendas prácticas sociales y colectivas tanto sus condiciones sociales de existencia como la naturaleza. En definitiva, el argumento refuerza el talante inequívocamente social y transformador de la actividad humana material e intelectual.

${ }^{3}$ En el enfoque estratégico-relacional de Jessop (1990, N.G. Sum y Jessop, 2015), los sistemas sociales autopoiéticos son analizados conforme a determinados criterios de la economía política crítica de Marx. Dicha conjunción brinda un potente marco conceptual para la teoría social, susceptible de dialogar con las contribuciones teóricas de A. Honneth. Sin embargo, en el presente artículo, no se abordan tales posibilidades analíticas.

${ }^{4}$ Niveles explicados en “Competencias de la población adulta en Chile: Resultados PIAAC”, MINEDUC, 2016.

${ }^{5}$ La teoría de la actividad histórico-cultural se ha desarrollado a través de "tres generaciones" de investigación. La "primera generación” es iniciada por Vygotsky en las décadas de 1920 y 1930, dando especial énfasis al concepto de "mediación cultural de las acciones". La "segunda generación" se presenta con Leontiev, poniéndose el acento en el reforzamiento analítico del estudio de la “actividad grupal” inmersa en un "sistema de actividad colectivo". Por último, la "tercera generación” se articula en torno a los planteamientos de Engeström, el cual profundiza el estudio de las relaciones existentes entre diferentes "sistemas de actividad" que comparten un objetivo común, y del aprendizaje que ocurre en su interior sobre la base de interacciones sistémicas (Engeström, 2001).
\end{abstract}

\section{Bibliografía}

BID (2018). El futuro del trabajo: Perspectivas regionales. Recuperado de https://publications.iadb.org/publications/ spanish/document/El-futuro-del-trabajo-Perspectivas-regionales.pdf

Block, F. (1990). Postindustrial Possibilities: A Critique of Economic Discourse. Berkeley, United States: University of California Press.

Block, F. (2011). Contesting markets all the way down. Journal of Australian Political Economy, (68), 27.

Block, F. y Evans, P. (2007). El Estado y la economía en Instituciones y desarrollo en la era de la globalización neoliberal. p. 307-350, Bogotá, Colombia: ILSA.

Block, F. y Somers, M. (2014). The Power of Market Fundamentalism. Karl Polanyi's Critique. Boston, United States: Harvard University Press.

Brunner, J. (2002). Educación: escenarios de futuro: nuevas tecnologías y sociedad de la información. Recuperado de https://educacion.udd.cl/files/2017/03/MS_Brunner-Educacion_escenarios_de_futuroNuevas_tecnologias_y_la_soc_info-1.pdf 
Busso, M., Bassi, M., Urzúa, S.,y Vargas, J. (2012). Desconectados: habilidades, educación y empleo en América Latina. New York, United States: Inter-American Development Bank.

Carneiro, P., y Heckman, J. (2003). Human capital policy. Izar Working Paper 821.

Curtis, D. (2006). Mind sets and methods: poverty strategies and the awkward potential of the enabling state. International Journal of Public Sector Management, 19(2), 150-164.

Dewey, J. (1988). Human nature and conduct. Southern Illinois. Carbondale, United States: University Press.

Dorf, M. C., y Sabel, C. F. (1998). A constitution of democratic experimentalism. Columbia law review, 267-473.

Edwards, S. (2010). Left behind: Latin America and the false promise of populism. Chicago, United States: University of Chicago Press.

Engeström, Y. (1987). Learning by expanding: An Activity Theoretical approach to developmental research. Helsinki, Finlandia: Orienta Konsultit.

Evans, K. (2000).Reclaiming John Dewey: Democracy, inquiry, pragmatism, and public management. Administration \& Society 32 (3), 308-328.

Evans, P. (1995), Embedded autonomy. Princeton, United States: Princeton University Press

Evans, P. (2007). Instituciones y desarrollo en la era de la globalización neoliberal, ILSA, Bogotá, Colombia.

Evans, P. (2008a). Is an alternative globalization possible? Politics \& Society, 36(2), 271-305.

Evans, P. (2008b), In search of the 21st century developmental state. The Centre for Global Political Economy, University of Sussex Working Paper, 4.

Falletti, T.G. y Parredo E.A. (Ed.) (2017) Latin America since the Left turn. Philadelphia, United States: University of Pennsylvania Press.

Gilbert, N.(2004). Transformation of the welfare state: The silent surrender of public responsibility. New York, United States: Oxford University Press.

Gilbert, N.(2005). The“Enabling State?” from Public to Private Responsibility for Social Protection. Working Paper, 26. Paris, France: OECD.

Good, J. (2005). A Search for Unity in Diversity: The Permanent Hegelian Deposit'in the Philosophy of John Dewey. Oxford, United Kingdom: Lexington Books.

Good, J. (2018). The German Bildung Tradition. UNC Charlotte Department of Philosophy. Recuperado de http:/ /www.philosophy.uncc.edu/mleldrid/SAAP/USC/pbt1.html

Gøsta Esping-Andersen (1990). The three worlds of welfare capitalism. Cambridge, United Kingdom:Princeton University Press.

Gøsta Esping-Andersen., Gallie, D., Hemerijck, A., \& Myles, J. (2002). Why we need a new welfare state. Oxford, United States:Oxford University Press.

Honneth, A. (2017). The idea of socialism. Towards a renewal. Cambridge, United Kingdom: Polity Press 
Jessop, B. (1990). State theory: Putting the capitalist state in its place. Cambridge, UK: Polity Press.

Kettunen, P. (2012). Hyvän Kehän Kerroksen ja rajat. En S. Alaja (ed.) Kriisikierteestä hyvän Kehään. Kalevi Sorsa-Säätiö 4/2012, pp.13-38.

Kristensen, y Lilja, K. (2011). The Co-evolution of Experimentalist Business Systems and Enabling Welfare States. Nordic capitalisms and globalization. En New forms of economic organization and welfare institutions, 1-46.Oxford, United States: Oxford University Press,

Miettinen, R. (2013). Innovation, Human capabilities, and Democracy: Towards an enabling welfare state. London, United Kingdom: Oxford University Press.

Miettinen, R. (2014). Information technological revolution and institutional innovations en Center for Research on Activity, Development and Learning. Working papers 4. Helsinki, Finland: University of Helsinki.

Miettinen, R. (2016). Sivistys kilpailuyhteiskunnassa ja Hegelin teorian vapauden ja minän kehityksen edellytyksistä”. Kasvatus ja Aika. 10 (3).

Mjoset, L. (Ed.) (2011). The Nordic varieties of capitalism. Bingley, United Kingdom:Emerald Group Publisher Limited.

OCDE (2017). Evaluaciones de Políticas Nacionales de Educación. Educación en Chile. Recuperado de https:// www.oecd-ilibrary.org/education/education-in-chile_9789264284425-en

Perez, C. (2002). Technological revolutions and financial capital: The dynamics of bubbles and golden ages.London, United Kingdom:Edward Elgar Publishing.

Polanyi, K. (2001). The Great Transformation. The political and economic origins of our time. New York, United States:Ameron House.

Sabel, C. F. (2006). A real-time revolution in routines. The firm as a collaborative community, 106, 110-13.

Sabel, C., Saxenian, A., Miettinen, R., Kristensen, P. H., \& Hautamäki, J. (2010). Individualized service provision in the new welfare state: Lessons from special education in Finland en SITRA Studies 62, Helsinki: SITRA.

Sader, E. (Coord.) (2016). Las Vías Abiertas de América Latina. Caracas, Venezuela:Octubre Editorial.

Sandbrook, R. (2014). Reinventing the Left in the Global South. The politics of the possible. Cambridge, United Kingdom: University Press. Cambridge

Sandbrook, R. y Edelman, M. (2007). Social democracy in the Global periphery: origins, challenges, prospects. Cambridge, United Kingdom: University Press. Cambridge

Sen, A. (1984). Resources, Values and Development. Oxford, United States: Basil Blackwell.

Sum, N. L., \& Jessop, B. (2015). Cultural political economy and critical policy studies: developing a critique of domination. EnHandbook of Critical Policy Studies, 128, Edward Elgar Publishing Limited.

Valsiner, J. (2000). Culture and Human Development. London, United Kingdom: Sage

Vygotsky, L. S. (1987). Thought and Language. Cambridge, United States:Harvard University Press. 